\title{
Lung transplantation in telomerase mutation carriers with pulmonary fibrosis
}

\author{
Leann L. Silhan', Pali D. Shah1, Daniel C. Chambers ${ }^{2,3}$, Laurie D. Snyder, \\ Gerdt C. Riise ${ }^{5}$, Christa L. Wagner ${ }^{6}$, Eva Hellström-Lindberg ${ }^{7}$, \\ Jonathan B. Orens ${ }^{1}$, Juliette F. Mewton ${ }^{8}$, Sonye K. Danoff ${ }^{1}$, Murat O. Arcasoy ${ }^{4}$ and \\ Mary Armanios 6,9
}

Affiliations: 'Dept of Medicine, Johns Hopkins University School of Medicine, Baltimore, MD, USA. ${ }^{2}$ Dept of Medicine, The Prince Charles Hospital, Brisbane, Australia. ${ }^{3}$ The University of Queensland, Queensland Lung Transplant Service, The Prince Charles Hospital Brisbane, Australia. ${ }^{4}$ Dept of Medicine, Duke University School of Medicine, Durham, NC, USA. ${ }^{5}$ Sahlgrenska University Hospital, Gothenburg, Sweden. ${ }^{6}$ Dept of Oncology, Johns Hopkins University School of Medicine, Baltimore, MD, USA. ${ }^{7}$ Dept of Medicine, Karolinska Institute, Huddinge, Sweden. ${ }^{8}$ The Prince Charles Hospital Renal Services, Brisbane, Australia. ${ }^{9}$ Sidney Kimmel Comprehensive Cancer Center, Johns Hopkins University School of Medicine, Baltimore, MD, USA.

Correspondence: Mary Armanios, 1650 Orleans St., Room 186, Baltimore, MD 21287, USA.

E-mail: marmani1ajhmi.edu

ABSTRACT Lung transplantation is the only intervention that prolongs survival in idiopathic pulmonary fibrosis (IPF). Telomerase mutations are the most common identifiable genetic cause of IPF, and at times, the telomere defect manifests in extrapulmonary disease such as bone marrow failure. The relevance of this genetic diagnosis for lung transplant management has not been examined.

We gathered an international series of telomerase mutation carriers who underwent lung transplant in the USA, Australia and Sweden.

The median age at transplant was 52 years. Seven recipients are alive with a median follow-up of 1.9 years (range 6 months to 9 years); one died at 10 months. The most common complications were haematological, with recipients requiring platelet transfusion support (88\%) and adjustment of immunosuppressives (100\%). Four recipients $(50 \%)$ required dialysis for tubular injury and calcineurin inhibitor toxicity. These complications occurred at significantly higher rates relative to historic series $(\mathrm{p}<0.0001)$.

Our observations support the feasibility of lung transplantation in telomerase mutation carriers; however, severe post-transplant complications reflecting the syndromic nature of their disease appear to occur at higher rates. While these findings need to be expanded to other cohorts, caution should be exercised when approaching the transplant evaluation and management of this subset of pulmonary fibrosis patients.

@ERSpublications

Telomerase mutation carriers with IPF may be prone to complications from their underlying telomere syndrome after LTx http://ow.ly/wmy6P

This article has supplementary material available from www.erj.ersjournals.com

Received: Feb 052014 | Accepted after revision: April 252014 | First published online: May 152014

Support statement: This work was supported by funding from the United States National Institutes of Health P30 CA006973 and the Flight Attendants Medical Research Institute (FAMRI).

Conflict of interest: Disclosures can be found alongside the online version of this article at www.erj.ersjournals.com

Copyright OERS 2014. ERJ Open articles are open access and distributed under the terms of the Creative Commons Attribution Non-Commercial Licence 3.0. 


\section{Introduction}

Idiopathic pulmonary fibrosis (IPF) is progressive and fatal, and lung transplantation is the only therapy that has been shown to prolong survival $[1,2]$. Because of recent changes in allocation algorithms, IPF has emerged as the leading indication, accounting for one-third of lung transplant cases [1,3-7]. Even though IPF remains defined by its idiopathic adjective, its most frequent identifiable genetic cause is inherited mutations in the telomerase genes [8]. Loss of function mutations in TERT, the telomerase reverse transcriptase gene, and TR (also known as TERC), the telomerase RNA, underlie up to $20 \%$ and $3 \%$ of familial and sporadic pulmonary fibrosis cases, respectively $[8,9]$. Mutations in the telomerase accessory component, $\mathrm{DKC1}$, the dyskeratosis congenita 1 gene, can also manifest as IPF, underscoring the important role of telomere dysfunction in IPF pathogenesis [10]. The significance of the genetic diagnosis of telomerase-mediated pulmonary fibrosis to lung transplant management has not been examined.

Mutations in telomerase cause its loss of function and mediate disease through abnormally shortened telomeres [11]. As such, the mutant gene and mutation type are not the primary mediators of disease severity but the telomere length defect, as previously reviewed [11]. Telomeres are DNA-protein structures that protect chromosome ends. With cell division and advancing age, telomeres shorten, and dysfunctional telomeres provoke cellular senescence and apoptosis [11]. IPF patients with telomerase mutations may show age-related cosmetic features such as premature hair greying, but these findings are not specific, and the diagnosis is confirmed by genetic and molecular testing [8]. Although lung disease is the predominant lifethreatening manifestation in adults with telomerase mutations, the telomere defect is systemic, and can be recognised in extrapulmonary manifestations that follow a stereotypical, syndromic pattern [12-14]. Bone marrow failure is the most frequent extrapulmonary manifestation and is often first detected as isolated thrombocytopenia in the setting of a hypocellular bone marrow [12-14]. Patients with telomere disorders are also prone to developing liver cirrhosis, enteropathies and osteoporosis, and have a higher incidence of skin and haematological malignancies $[11,15,16]$. Even in the absence of telomerase mutations, IPF patients can have abnormally short telomeres $[17,18]$ and can manifest syndromic features of telomeremediated disease [18]. These observations have suggested telomere defects may be relevant to pathogenesis even beyond the IPF subset with telomerase mutations [18].

We sought here to examine the relevance of the telomere syndrome diagnosis in IPF patients who underwent lung transplantation. This question is of particular significance because bone marrow failure patients with telomere syndromes are exquisitely sensitive to standard bone marrow transplant conditioning regimen, as previously reviewed $[19,20]$. These observations have contributed to the current standard of care practice of reducing the intensity of the bone marrow transplant conditioning regimens with significantly improved short-term outcomes [8]. Therefore, pre-transplant screening for telomere syndromes in patients with bone marrow failure has been advocated and is being integrated into standard work-ups since it significantly alters the bone marrow transplant approach [8]. In this study, we report a first and international series of lung transplant experience in pulmonary fibrosis patients who carry mutant telomerase genes. We show that adult telomerase mutation carriers with IPF who undergo lung transplant develop recurrent and otherwise unusual complications that reflect the syndromic nature of their disease. Our observations suggest that recognising these patients, clinically and through genetic evaluation, may inform management in this subset of lung transplant recipients.

\section{Subjects and methods}

Subjects were eligible for this study if they carried a mutation in telomerase, or had the diagnosis of a telomere syndrome and had received a lung transplant. Telomere syndrome criteria were defined as previously, and confirmed by abnormally short age-adjusted telomere length [13, 14, 18]. Cases were identified through genetic evaluation at Johns Hopkins, Baltimore, MD, USA and through a collaborative effort of a list-serve for lung transplant physicians from around the world. Extracted and primary medical records were reviewed by two of the authors. The study was approved by the Johns Hopkins Medicine institutional review board, and the human research and ethics committees of contributing institutions. Subjects recruited within the USA gave written, informed consent. Follow-up was updated on March 1, 2014.

To determine the incidence of medication-related toxicities in telomerase mutation carriers, we reviewed the Johns Hopkins experience and the literature for adverse events of commonly prescribed drugs in the lung transplant setting: azathioprine, calcineurin inhibitors, de novo purine synthesis antagonists and antibiotics. Events recorded from five additional pulmonary fibrosis cases enrolled in the Johns Hopkins Telomere Syndrome Registry who received at least one of these medication classes in other settings along with events extracted from a literature review of telomerase mutation carriers with pulmonary disease are included. Details of the manual literature review (through December 31, 2012) have been previously 
published [15]. We used GraphPad Prism software for statistical analyses (San Diego, CA, USA). The p-values shown are all two-sided.

\section{Results}

\section{Lung transplant recipients have clinical features of a telomere syndrome}

The eight subjects were transplanted at four centres from 2004 to 2013 in the USA $(n=5)$, Australia $(n=2)$, and Sweden $(n=1)$. The median age at pulmonary fibrosis diagnosis was 47 years (range $42-61$ years) and 50\% were male. The median age at transplant was 52 years (range 44-64 years). Most subjects showed features of a telomere syndrome prior to transplant, including premature hair greying prior to 25 years of age (six of seven; $86 \%$ ) and abnormally low blood counts with at least one haematopoietic lineage affected (thrombocytopenia most common, five of eight; 63\%). One subject carried the diagnosis of myelodysplastic syndrome, and one had bone marrow failure. Three subjects (38\%) had history of resection of squamous or basal cell skin carcinomas. All subjects with available family histories reported having at least one relative with pulmonary fibrosis (six of six; 100\%). All subjects had documented normal renal function prior to transplant. The pre-transplant clinical characteristics are summarised in table 1.

\section{Molecular studies support the telomere syndrome diagnosis}

The genetic diagnosis was documented prior to transplant in half the cases. Five subjects carried mutations in TERT, two subjects had mutations in $T R$, and one fulfilled clinical telomere syndrome criteria with abnormally short telomere length, a positive family history of pulmonary fibrosis, a personal history of thrombocytopenia but no detectable mutation in TERT, TR or DKC1. The TERT and TR mutations identified were absent in large series of controls ( $n=1500$ including the 1000 Genome Project [21]) and fell in highly conserved motifs (fig. 1 and supplementary fig. S1). Four of the mutations were previously reported in telomere disorders or shown to functionally decrease telomerase activity [14, 22, 23]. Where available (five of five), telomere length by flow cytometry and FISH fell below the age-adjusted first percentile, supporting the pathogenic nature of these mutations (fig. 2a). Since the majority of the cases in this series $(88 \%)$ carry telomerase mutations, similar to the documented literature [14], we will use the terminology telomerase mutation carriers to refer to this cohort hereafter.

\section{Lung transplant outcomes in telomerase mutation carriers}

Seven of the eight subjects $(88 \%)$ are alive with a median follow-up of 1.9 years (range 6 months to 9 years). One subject died 10 months post-transplant after a prolonged hospitalisation. The post-transplant clinical course is summarised in table 2 and in figure 2b-h. Explanted lung pathology documented usual interstitial pneumonia changes in all cases. All the subjects underwent at least two transbronchial biopsies post-transplant. Four had no rejection and four had evidence of minimal rejection (grade A1 B0, 50\%, International Society for Heart and Lung Transplantation classification [24]), and one of these had transient evidence of moderate rejection (grade A2) rejection. Several subjects suffered infectious complications with Gram negative pneumonia/sepsis and opportunistic infections, such as pulmonary aspergillosis and cytomegalovirus pneumonitis (fig. $2 \mathrm{c}$ and d). One subject had defective healing at the anastomotic site bilaterally with each of sequential lung transplants; delayed wound healing has been reported in other telomere syndrome cases [10].

\section{Increased rate of haematological complications post-transplant}

Haematological complications were the most prevalent morbidity and required significant adjustment of the immunosuppressive regimen in all cases (100\%). Most subjects were able to tolerate only two drug immunosuppressive regimen. A majority of recipients required transfusion support for thrombocytopenia (seven of eight, 88\%; fig. 2b), and several required bone marrow evaluation and formal haematology consultation for concerns regarding the severity of thrombocytopenia and risk of bleeding. In a large historical series of 169 patients, a 12\% platelet transfusion rate was documented after lung transplantation [25]; therefore, the transfusion need in this international cohort of short telomere patients appears significantly increased $(\mathrm{p}<0.0001$; Chi-squared test $)$. Platelet counts improved with adjustment of myelosuppressive medications but remained lower than pre-transplant levels, and four subjects, including the two cases that had a bone marrow failure diagnosis prior to lung transplant continue to require platelet transfusion prophylactically prior to invasive procedures. One of these individuals also developed haemolytic anaemia and remains red blood cell transfusion-dependent 9 months after transplant (table 2).

\section{High frequency of renal failure requiring RRT in telomerase mutation carriers}

In addition to the haematological complications, there was also a surprisingly high rate of acute renal failure and acute tubular necrosis. Four individuals required renal replacement therapy (RRT) in the first 2 weeks post-transplant (50\%). Relative to historical series where RRT is indicated in $0.6 \%-4.5 \%$ in the first month 


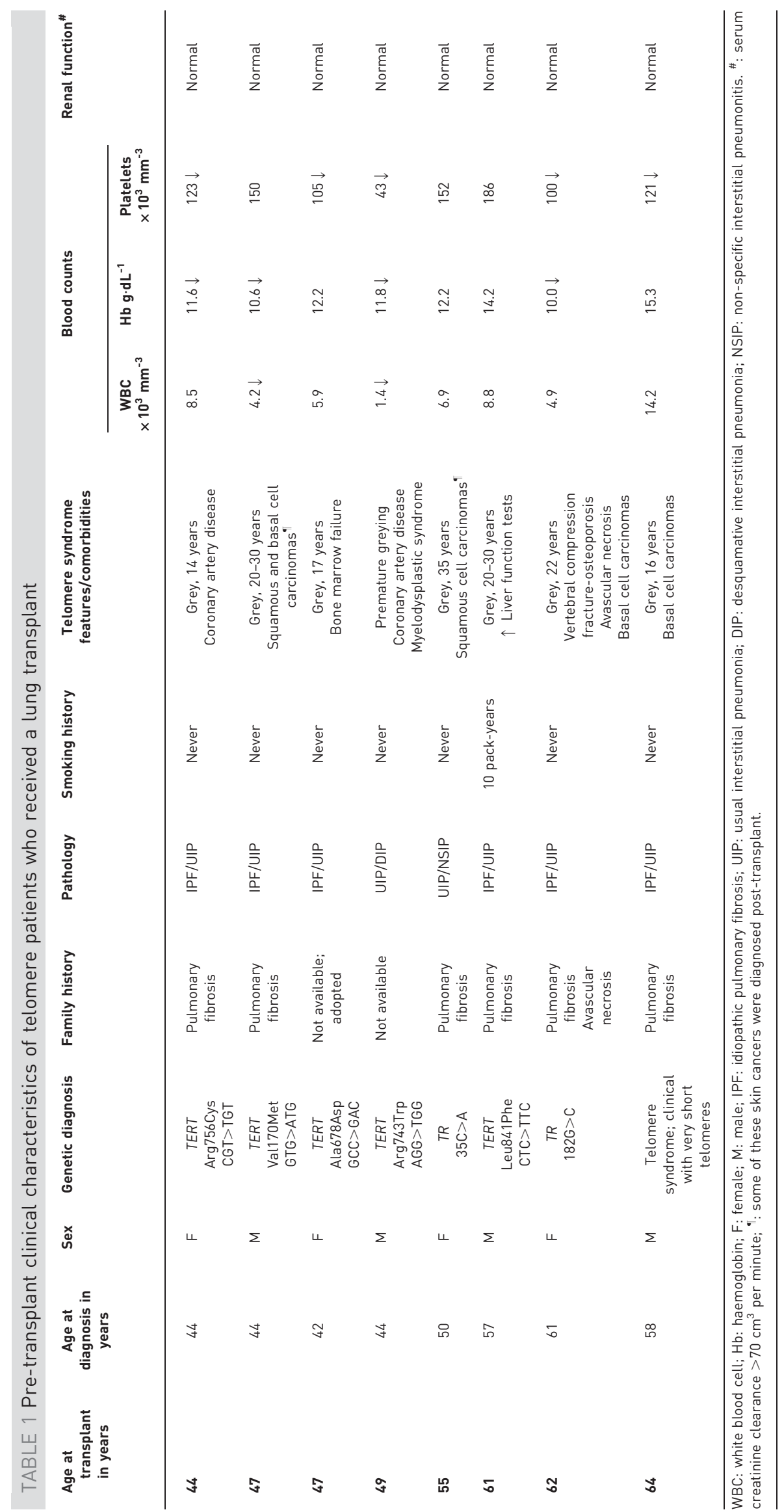


a)

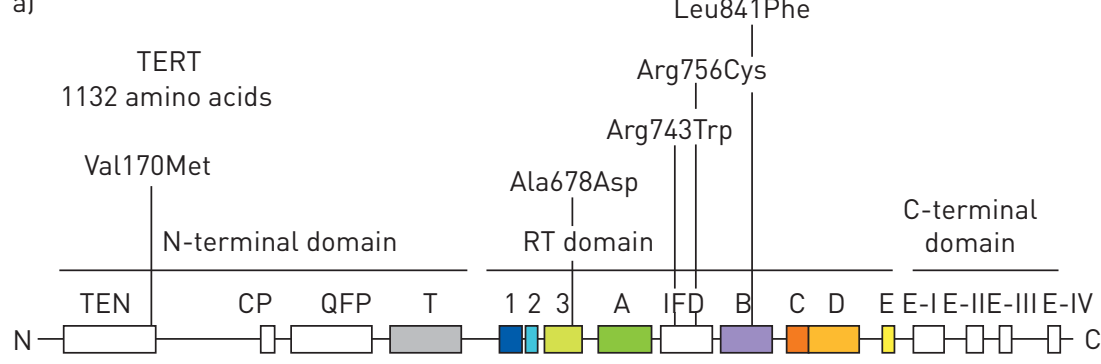

b)

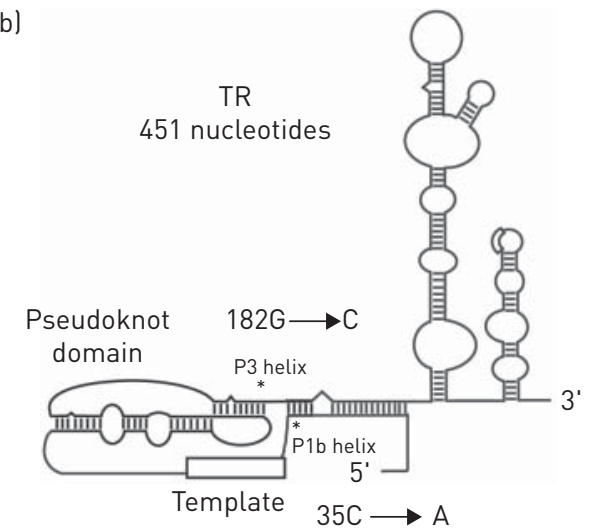

FIGURE 1 Telomerase mutations in lung transplant subjects fall in conserved domains of telomerase reverse transcriptase (TERT) and telomerase RNA (TR). a) Organisation of conserved reverse transcriptase motifs (boxed) with mutations indicated above $(n=5)$. b) TR mutations fall in the P1b and P3 helices within the secondary structure and are near the template-containing pseudoknot domain. Both are predicted to disrupt Watson-Crick base pairing and thus TR stability.

after lung transplant $[26,27]$, this frequency is unusually high $(\mathrm{p}<0.0001$; Chi-squared test). The renal failure was thought to be multi-factorial related to perioperative haemodynamic changes, infections, and calcineurin inhibitor nephrotoxicity. One subject underwent a renal transplant after 8 months, while the other three recovered after 9 months, 2 months and 8 days of RRT, but all continue to have residual chronic renal insufficiency. In addition, the 9-year survivor has developed end stage renal disease. Renal histopathology from two cases that required prolonged dialysis was available, and showed similar findings of tubular epithelial irregularity, sloughing, and proximal tubule cytoplasmic vacuolisation (fig. 2e and f). These findings are hallmarks of calcineurin inhibitor toxicity [28]. Calcineurin-inhibitor nephrotoxicity occurs in $25 \%$ of lung transplant recipients at 1 year, but only $2-4 \%$ of cases require RRT, suggesting a higher rate of toxicity in the group of patients we studied $(p=0.008)[26,29]$.

In addition to haematological and renal complications, three individuals developed gastrointestinal bleeding that required red blood cell transfusion support (three of eight, 37\%). In one case, the haemorrhage was caused by mycophenolate-related ischaemic colitis, a very rare complication limited to case reports [30, 31]. Colon biopsy after discontinuation of mycophenolate showed residual crypt loss and epithelial apoptosis similar to histopathological findings documented in telomere-related enteropathy (fig. $2 \mathrm{~g}$ and $\mathrm{h}$ ) [15]. In some cases there was impaired functional recovery with three individuals developing critical illness polyneuropathy and myopathy; in two cases, this was deemed severe and contributed to prolonged hospitalisation beyond 3 months.

\section{Patients with telomere defects are at risk for rare medication-related toxicities}

In addition to mycophenolate and calcineurin inhibitors, lung transplant recipients are exposed to a number of cytotoxic medications as part of the immunosuppression or anti-microbial prophylaxis regimens. Because there is precedence for increased adverse events with medications in patients with telomere disorders (such as busulfan in the bone marrow transplant setting where it causes fatal pulmonary fibrosis [19]), we extended our search to individuals in the Johns Hopkins Telomere Syndrome Registry and the literature to identify serious toxicities that may also be relevant to lung transplant recipients. We identified adverse events with azathioprine, dapsone and inhaled pentamidine. The two azathioprine-related toxicities manifested as fulminant hepatitis, one of which was fatal [12, 32]. Azathioprine-related fulminant hepatitis occurs in less than one in 10000 cases [33], making these two reports in a small subset highly noteworthy. We also observed methemoglobinaemia with short-term dapsone use, and a first case to our knowledge of transient hypoglycaemia after aerosolised pentamidine, a complication that rarely occurs even with systemic administration [34, 35].

\section{Discussion}

In this international series, we report that lung transplantation for IPF patients with telomerase mutations is feasible, and can at times be associated with reasonable survival. The longest surviving case in our series is nine years post-transplant. Nevertheless, our experience suggests that transplantation in this group of patients requires attention to specific complications that may otherwise be rare in lung transplant recipients and that at times can be severe. These include haematological toxicities with otherwise routinely used medications, and in many cases, at least a short-term requirement for transfusion support for significant 

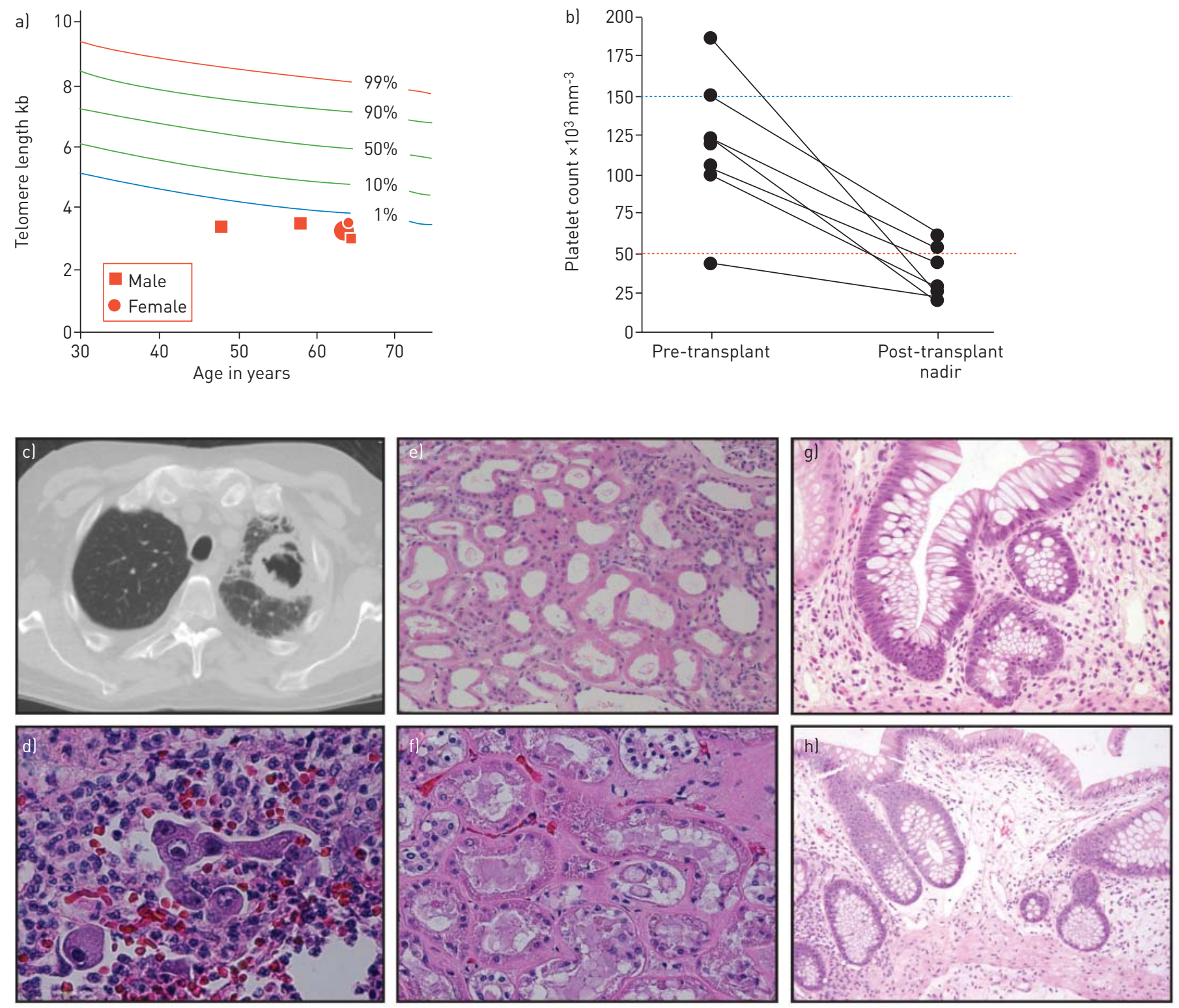

FIGURE 2 Post-transplant complications of telomere syndrome cases. a) Telomere length by flow cytometry and fluorescence in situ hybridisation shows significant shortening relative to age-matched controls ( TERT, $\mathrm{n}=2 ; T R, \mathrm{n}=2$; clinical telomere syndrome $\mathrm{n}=1$ ). The telomere length nomogram is based on 400 controls. b) Pre-transplant platelet counts show a majority of patients had thrombocytopenia $\left(<150 \times 10^{3}\right.$ platelets $\left.\mathrm{mm}^{-3}\right)$. Post-transplant, platelet counts drop further and in several cases into a range that increases the risk of bleeding $\left(<50 \times 10^{3}\right.$ platelets $\left.\mathrm{mm}^{-3}\right)$. Post-transplant nadir is defined as within the first two weeks. c) Computed tomography image showing cavitary left lung lesion in a patient with pulmonary aspergillosis at 9 months post-transplant. d) Lung histopathology from autopsy in a subject who died from complications of cytomegalovirus pneumonitis. The photomicrograph shows evidence of diffuse alveolar haemorrhage and multiple viral intranuclear inclusions. e and f) Renal biopsies of two individuals who required prolonged dialysis showing tubular irregularity and epithelial cytoplasmic vacuolisation; these findings are highly consistent with calcineurin inhibitor toxicitiy. Biopsies were obtained at 4 months (e) and 10 months (f) post-dialysis, the latter at the time of autopsy. g) Colon biopsy from individual who developed ischaemic colitis on mycophenolate shows extensive puss, crypt dropout and apoptosis. h) Colon biopsy from the same individual 5 weeks after mycophenolate was discontinued shows resolution of the puss, but residual crypt dropout and epithelial apoptosis similar to histological findings described in other telomere syndrome cases and consistent with an underlying telomere-mediated enteropathy.

cytopenias. Awareness of these potential complications may help anticipate, manage and possibly avert post-transplant morbidities in this IPF subset.

Recipients in our series uniformly required dose modification of immunosuppression for cytopenias, reflecting their underlying limited bone marrow reserves. Platelet transfusion support was also necessary and, in some cases, this need was prolonged. Pre-transplant challenge protocols with cytotoxic drugs are used in some experienced centres to test the robustness of bone marrow reserves, and this may be one approach to identify patients who can tolerate the haematological stress of transplant-related 


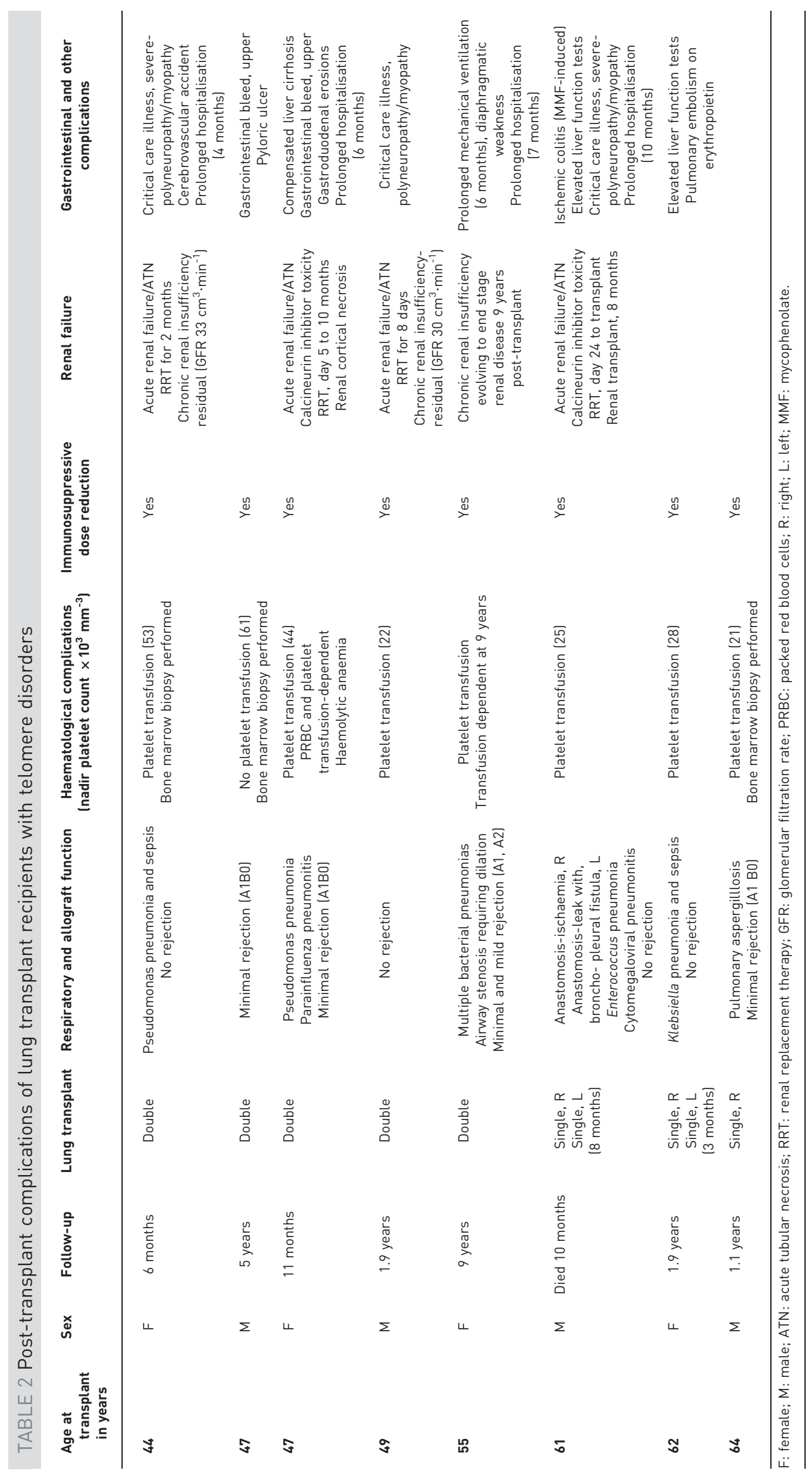


myelosuppressive medications. In our series, one patient who carried the diagnosis of myelodysplastic syndrome and one patient who had bone marrow failure received a lung transplant; the former patient is doing well 1.9 years out from transplant and is transfusion independent, while the latter remained transfusion-dependent 11 months post-transplant. These cases highlight the heterogeneity in the severity of bone marrow failure in telomere syndrome patients and the need for multidisciplinary consultation with haematologists in assessing bone marrow function. The clinical criteria, based on medical and family history, for suspecting the telomere syndrome diagnosis along with considerations for a telomere-focused pre-transplant work-up are summarised in figure 3.

Our experience suggests it is possible that nephrotoxic drugs used in the transplant setting may uncover a telomere-related renal vulnerability. Despite the fact that all the recipients had normal renal function pretransplant, four cases $(50 \%)$ required RRT and, in a subset of these cases, the need was prolonged. Renal disease is not known to occur de novo in telomere syndromes, and has been reported only once after bone marrow transplantation [36]. Calcineurin inhibitors levels are maintained at higher levels in lung transplant recipients because of the relatively high incidence of allograft rejection [37, 38]; this may contribute to uncovering a telomere-related renal vulnerability. Calcineurin inhibitors are also metabolised by hepatic P450 enzymes [39], and it is possible that patients who have subclinical liver disease are prone to decreased clearance. Indeed, in two cases that required prolonged RRT, there was abnormal liver function and evidence of subclinical cirrhosis radiographically (table 2). It is interesting to note that mice with short telomere are prone to renal tubular apoptosis and have an increased predilection to tubular epithelial injury after challenge [40]. Perioperative renal compromise, along with the nephrotoxic effects of calcineurin inhibitors may thus cause a greater than expected tubular injury which may be difficult to reverse because of the telomere defect. One compelling possibility is that telomere syndrome patients may have a lower frequency of allograft rejection because of an immunosenescence phenotype. This hypothesis will need to be tested prospectively and if supported, it may provide a rationale for modifying the current immunosuppressive regimens for this population. Understanding the spectrum of toxicities associated with calcineurin inhibitors in telomere syndrome patients will also be important in informing the optimal approach to immunosuppression in the lung transplant setting.

Despite the small nature of our series, the concordant findings across four centres from around the world support that this group of patients may warrant specific attention to complications that may otherwise be uncommon in other lung recipient populations, such as cystic fibrosis or pulmonary hypertension. In patients with telomere syndromes who require bone marrow transplantation, the experience of recent decades has led to a significant alteration in the approach to bone marrow transplant with improved shortterm outcomes $[19,20]$. For example, it is considered standard practice to shield the lungs during radiation exposure and to minimise DNA damaging agents [20]. Therefore, in the context of an existing individualised paradigm for bone marrow transplant in these patients, our observations suggest that a similar tailored lung transplant protocol may be necessary to optimise supportive care for this subset of pulmonary fibrosis patients.

a) Clinical features that heighten suspicion for telomere syndrome diagnosis

Family history of pulmonary fibrosis and/or bone marrow failure

Premature greying (before age 25 years)

Haematological abnormalities (e.g. thrombocytopenia, macrocytosis)

Liver disease or abnormal liver function

History of defective wound healing

History of non-melanoma skin cancer (squamous, basal cell carcinoma) b) Pre-transplant work-up considerations for patients suspected to have telomere syndrome diagnosis

Consider genetic counselling and evaluation

Consider formal haematology consultation

Consult with experienced centre regarding feasibility of bone marrow challenge

Assess liver parenchyma for subclinical evidence of cirrhosis (e.g. with ultrasound)

If indicated, counsel patient regarding risk of telomere-specific complications (e.g. haematological)

FIGURE 3 Clinical considerations for a) suspecting and b) evaluating patients with pulmonary fibrosis who may have telomere syndromes. 
Finally, it is important to note that our series reflects only cases that were deemed eligible for transplantation. Transplant candidates with pulmonary fibrosis and concurrent end organ failure in the bone marrow and liver were frequently deemed ineligible at all of our centres. Moreover, despite the specificity of the IPF-bone marrow failure complex for telomere defects, the majority of IPF patients with telomerase mutations cannot be distinguished clinically because they have disease that appears limited to the lung, and these individuals would not have been selected for genetic evaluation [14, 41, 42]. The spectrum of lung disease pathology associated with telomerase mutations is also broad, and although the IPF/usual interstitial pneumonia phenotype is most common in telomerase mutation carriers, in up to $30 \%$ of cases, the clinical picture may reflect another idiopathic interstitial pneumonia, as reviewed elsewhere [43]. Pre-transplant identification may facilitate risk assessment and inform post-transplant management for this subset. Importantly, because the telomere defect is commonly seen in IPF patients even in the absence of telomerase mutations [18, 44], our experience raises the possibility that syndromic defects may contribute to the inferior post-transplant outcomes in IPF relative to other lung transplant recipients. These hypotheses are testable and, if validated, may form the basis of a genetically informed transplant management paradigm for a subset of pulmonary fibrosis patients.

\section{Acknowledgements}

We are grateful to all the subjects and their treating clinicians. We are grateful to Dr. Soheir Adam for case referrals, Dr. Elizabeth Montgomery, Dr. Emily Nelson and Dr. Joanna Perry-Keene for the histopathology images, Dr. Naudia Lauder Jonassaint, Dr. Jonathan F. McDyer, Dr. Nada Alachkar, and the Duke University Pulmonary Transplant team for helpful discussions. We appreciate critical comments on the manuscript from Dr. Paul Hassoun and Dr. Robert A. Wise.

\section{References}

King TE Jr, Pardo A, Selman M. Idiopathic pulmonary fibrosis. Lancet 2011; 378: 1949-1961.

Richeldi L, Collard HR, du Bois RM, et al. Mapping the future for pulmonary fibrosis: report from the 17th International Colloquium on Lung and Airway Fibrosis. Eur Respir J 2013; 42: 230-238.

3 Thabut G, Mal H, Castier Y, et al. Survival benefit of lung transplantation for patients with idiopathic pulmonary fibrosis. J Thorac Cardiovasc Surg 2003; 126: 469-475.

4 George TJ, Arnaoutakis GJ, Shah AS. Lung transplant in idiopathic pulmonary fibrosis. Arch Surg 2011; 146: 1204-1209.

5 Christie JD, Edwards LB, Kucheryavaya AY, et al. The Registry of the International Society for Heart and Lung Transplantation: 29th adult lung and heart-lung transplant report-2012. J Heart Lung Transplant 2012; 31: 1073-1086.

6 Egan TM, Murray S, Bustami RT, et al. Development of the new lung allocation system in the United States. Am J Transplant 2006; 6: 1212-1227.

7 Algar FJ, Espinosa D, Moreno P, et al. Results of lung transplantation in idiopathic pulmonary fibrosis patients. Transplant Proc 2010; 42: 3211-3213.

8 Armanios M. Telomeres and age-related disease: how telomere biology informs clinical paradigms. J Clin Invest 2013; 123: 996-1002.

9 Garcia CK. Idiopathic pulmonary fibrosis: update on genetic discoveries. Proc Am Thorac Soc 2011; 8: 158-162.

10 Alder JK, Parry EM, Yegnasubramanian S, et al. Telomere phenotypes in females with heterozygous mutations in the dyskeratosis congenita 1 (DKC1) gene. Hum Mutat 2013; 34: 1481-1485. Armanios M, Blackburn EH. The telomere syndromes. Nat Rev Genet 2012; 13: 693-704.

12 Armanios M, Chen JL, Chang YP, et al. Haploinsufficiency of telomerase reverse transcriptase leads to anticipation in autosomal dominant dyskeratosis congenita. Proc Natl Acad Sci USA 2005; 102: 15960-15964.

13 Armanios M. Syndromes of telomere shortening. Annu Rev Genomics Hum Genet 2009; 10: 45-61.

14 Parry EM, Alder JK, Qi X, et al. Syndrome complex of bone marrow failure and pulmonary fibrosis predicts germline defects in telomerase. Blood 2011; 117: 5607-5611.

15 Jonassaint NL, Guo N, Califano JA, et al. The gastrointestinal manifestations of telomere-mediated disease. Aging Cell 2013; 12: 319-323.

16 Diaz de Leon A, Cronkhite JT, Katzenstein AL, et al. Telomere lengths, pulmonary fibrosis and telomerase (TERT) mutations. PLoS One 2010; 5: e10680.

17 Cronkhite JT, Xing C, Raghu G, et al. Telomere shortening in familial and sporadic pulmonary fibrosis. Am J Respir Crit Care Med 2008; 178: 729-737.

18 Alder JK, Chen JJ, Lancaster L, et al. Short telomeres are a risk factor for idiopathic pulmonary fibrosis. Proc Natl Acad Sci USA 2008; 105: 13051-13056.

19 de la Fuente J, Dokal I. Dyskeratosis congenita: advances in the understanding of the telomerase defect and the role of stem cell transplantation. Pediatr Transplant 2007; 11: 584-594.

20 Dietz AC, Orchard PJ, Baker KS, et al. Disease-specific hematopoietic cell transplantation: nonmyeloablative conditioning regimen for dyskeratosis congenita. Bone Marrow Transplant 2011; 46: 98-104.

21 Abecasis GR, Altshuler D, Auton A, et al. A map of human genome variation from population-scale sequencing. Nature 2010; 467: 1061-1073.

22 Chambers DC, Clarke BE, McGaughran J, et al. Lung fibrosis, premature graying, and macrocytosis. Am J Respir Crit Care Med 2012; 186: e8-e9.

23 Du HY, Pumbo E, Ivanovich J, et al. TERC and TERT gene mutations in patients with bone marrow failure and the significance of telomere length measurements. Blood 2009; 113: 309-316.

24 Stewart S, Fishbein MC, Snell GI, et al. Revision of the 1996 working formulation for the standardization of nomenclature in the diagnosis of lung rejection. J Heart Lung Transplant 2007; 26: 1229-1242. 

transfusion on outcome after lung transplantation. Eur J Cardiothorac Surg 2011; 39: 538-542.

DP, Solovera-Rozas M, Feng J, et al. Dialysis after lung transplantation: prevalence, risk factors and outcome. J Heart Lung Transplant 2007; 26: 1155-1162.

27 Hennessy SA, Gillen JR, Hranjec T, et al. Influence of hemodialysis on clinical outcomes after lung transplantation. J Surg Res 2013; 183: 916-921.

28 Liptak P, Ivanyi B. Primer: histopathology of calcineurin-inhibitor toxicity in renal allografts. Nat Clin Prac Nephrol 2006; 2: 398-404.

29 George TJ, Arnaoutakis GJ, Beaty CA, et al. Acute kidney injury increases mortality after lung transplantation. Ann Thorac Surg 2012; 94: 185-192.

30 Kim HC, Park SB. Mycophenolate mofetil-induced ischemic colitis. Transplant Proc 2000; 32: $1896-1897$.

31 Behrend M. Adverse gastrointestinal effects of mycophenolate mofetil: aetiology, incidence and management. Drug Saf 2001; 24: 645-663.

32 Calado RT, Regal JA, Kleiner DE, et al. A spectrum of severe familial liver disorders associate with telomerase mutations. PLOS ONE 2009; 4: e7926.

33 Gisbert JP, Gonzalez-Lama Y, Mate J. Thiopurine-induced liver injury in patients with inflammatory bowel disease: a systematic review. Am J Gastroenterol 2007; 102: 1518-1527.

34 Esbenshade AJ, Ho RH, Shintani A, et al. Dapsone-induced methemoglobinemia: a dose-related occurrence? Cancer 2011; 117: 3485-3492.

35 Bozzette SA, Finkelstein DM, Spector SA, et al. A randomized trial of three antipneumocystis agents in patients with advanced human immunodeficiency virus infection. NIAID AIDS Clinical Trials Group. N Engl J Med 1995; 332: 693-699.

36 Rocha V, Devergie A, Socie G, et al. Unusual complications after bone marrow transplantation for dyskeratosis congenita. Br J Haematol 1998; 103: 243-248.

37 Treede H, Glanville AR, Klepetko W, et al. Tacrolimus and cyclosporine have differential effects on the risk of development of bronchiolitis obliterans syndrome: results of a prospective, randomized international trial in lung transplantation. J Heart Lung Transplant 2012; 31: 797-804.

38 Baughman RP, Meyer KC, Nathanson I, et al. Monitoring of nonsteroidal immunosuppressive drugs in patients with lung disease and lung transplant recipients: American College of Chest Physicians evidence-based clinical practice guidelines. Chest 2012; 142: e1S-e111S.

39 Saeki T, Ueda K, Tanigawara Y, et al. Human P-glycoprotein transports cyclosporin A and FK506. J Biol Chem 1993; 268: 6077-6080.

40 Westhoff JH, Schildhorn C, Jacobi C, et al. Telomere shortening reduces regenerative capacity after acute kidney injury. J Am Soc Nephrol 2010; 21: 327-336.

41 Armanios MY, Chen JJ, Cogan JD, et al. Telomerase mutations in families with idiopathic pulmonary fibrosis. N Engl J Med 2007; 356: 1317-1326.

42 Alder JK, Cogan JD, Brown AF, et al. Ancestral mutation in telomerase causes defects in repeat addition processivity and manifests as familial pulmonary fibrosis. PLoS Genet 2011; 7: e1001352.

43 Armanios M. Telomerase and idiopathic pulmonary fibrosis. Mutation Res 2012; 730: 52-58.

44 Fukuhara A, Tanino Y, Ishii T, et al. Pulmonary fibrosis in dyskeratosis congenita with TINF2 gene mutation. Eur Respir J 2013; 42: 1757-1759. 